Service social

\title{
Le parallèle structurel entre la situation des jeunes LGBT et celle des organismes les desservant : quelques constats pour l'intervention
}

\section{Bruno Laprade}

Volume 59, numéro 1, 2013

L'homophobie et les tentatives de suicide et la résilience chez les jeunes LGBTQ

URI : https://id.erudit.org/iderudit/1017481ar

DOI : https://doi.org/10.7202/1017481ar

Aller au sommaire du numéro

Éditeur(s)

École de service social de l’Université Laval

ISSN

1708-1734 (numérique)

Découvrir la revue

Citer cet article

Laprade, B. (2013). Le parallèle structurel entre la situation des jeunes LGBT et celle des organismes les desservant : quelques constats pour l'intervention.

Service social, 59(1), 95-103. https://doi.org/10.7202/1017481ar d'utilisation que vous pouvez consulter en ligne. 


\section{Le parallèle structurel entre la situation des jeunes LGBT et celle des organismes les desservant : quelques constats pour l'intervention}

Bruno Laprade

Lors des entrevues d'embauche à Projet 10, on pose systématiquement la question : « Quels sont les défis rencontrés par les jeunes LGBT? » La réponse que l'on recherche de la part des candidats, au-delà des mentions du taux de suicide, de l'intimidation ou de la sortie du placard, est simple : les jeunes LGBT sont comme tous les jeunes, ils sont à la recherche de leur identité et de leur autonomie. Au Projet 10, on croit fortement à la capacité des jeunes à contrôler leur vie et à prendre leurs propres décisions. Cependant, de nombreuses barrières viennent limiter leurs possibilités d'action. L'âgisme à l'égard des jeunes est sans doute une des dernières discriminations systémiques encore acceptées dans notre société. À preuve, il existe non seulement un discours de délégitimation de la jeunesse (quand, par exemple, en entrevue Pauline Marois dit que Léo Bureau-Blouin, l'ancien porte-parole étudiant, est trop jeune pour devenir ministre), mais également des institutions juridiques et sociales chargée de lui reconnaître - ou non - son autonomie : pouvoir consentir à recevoir des actes médicaux (14 ans), consentir à des actes sexuels ou obtenir un permis de conduire (16 ans), droit de vote, droit de boire et de fumer (18 ans), etc. Quand bien même un jeune aurait acquis tous ces «droits » en atteignant la majorité, les effets de l'âgisme continueront de se faire sentir. On accordera davantage de crédibilité à un homme de 40 ans en veston cravate qu'à un jeune de 20 ans. Et les discours qui visent à « protéger les jeunes » ne sont souvent que des moyens de les contrôler ou de se faire du capital politique sur leur dos. Comme lorsque le gouvernement Harper a rehaussé l'âge de consentement à des actes sexuels. C'est ainsi que l'âgisme a certainement des impacts sur le développement des organismes jeunesse, et plus particulièrement sur ceux qui cherchent à leur laisser une place pour s'exprimer sans leur imposer un programme de santé publique ${ }^{1}$. J'aimerais m'attarder dans un premier temps à la place laissée à la parole des jeunes, notamment en ce qui a trait aux lieux où ils peuvent s'exprimer. À partir de l'histoire de deux organismes voués aux jeunes, je montrerai comment leur situation est similaire à l'isolement que vivent les jeunes eux-mêmes. À partir de mon expérience, je tirerai par la suite quelques constats quant aux capacités d'intervention du réseau de la santé dans les conditions actuelles.

Bien sûr, il faut s'entendre ici sur une définition de jeune. Le concept en lui-même est flou. Projet 10 et Jeunesse Lambda acceptent les personnes jusqu'à 25 ans. Pour le Forum jeunesse de l'île de Montréal, un jeune a en dessous de 35 ans. Pour plusieurs programmes de loisirs municipaux, ils ont entre 12 et 17 ans. Cette variation a sûrement son importance puisqu'une personne de moins de 18 ans a généralement bien moins d'autonomie mais cela ne se résout pas magiquement le jour où elle quitte le foyer familial.

1. Par là j'entends tous ces programmes pour lutter contre le tabagisme, l'obésité ou les infections transmissibles sexuellement ou par le sang (ITSS). 
En 2010, les États-Unis et le Canada² furent choqués par une vague de suicide chez des jeunes victimes d'intimidation. La plupart d'entre eux (et elles) étaient gai(e)s. Certains ont même laissé des messages sur leur blogue. Les médias s'en sont emparé et l'on a vu une vague de sympathie pour la cause, menant notamment à la création du projet It gets better. Des milliers de vidéos de soutien ont afflué, notamment de personnalités célèbres telles que le président Obama. Le message principal de It gets better est de demander aux jeunes de la diversité sexuelle de s'accrocher à la vie, de passer à travers la période difficile de l'adolescence (et son corollaire de l'école secondaire) puisque la vie s'améliore et qu'un jour, quand ils ne seront plus entourés d'homophobes, ils seront épanouis et heureux. Évidemment, de nombreuses critiques ont émergé pour souligner qu'il ne fallait pas attendre pour agir sur cette situation. L'une des plus intéressantes est sans doute Reteaching Gender \& Sexuality ${ }^{3}$, une vidéo faite par des jeunes LGBT dans le but d'éduquer leurs professeurs (et autres professionnels) sur leurs réalités. Leur propos souligne les contradictions de la campagne It gets better, l'intérêt médiatique soulevée par la vague de suicides, mais, surtout, il remet en cause la façon de percevoir le coming-out. Quoiqu'il soit souvent perçu comme une simple étape à franchir, ces jeunes rappellent que le coming-out se fait à tous les jours, de façon répétée ${ }^{4}$. C'est pourquoi «Reteaching Gender \& Sexuality intends to steer the conversation beyond the symptom of bullying, to consider systemic issues and deeper beliefs about gender and sexuality that impact queer youth ». Car la question demeure : pourquoi est-ce que la voix de ces jeunes qui se sont enlevé la vie nous est-elle arrivée si tard?

Après tout, l'enjeu du suicide chez les jeunes LGBT n'est pas nouveau. La littérature scientifique a un large éventail de recherches sur la vulnérabilité de cette population: décrochage scolaire, risque de consommation abusive d'alcool et de drogue, risque de grossesse plus élevé, taux de dépression plus élevé, etc. Le rapport de la Commission des droits de la personne et des droits de la jeunesse (CDPDJ) relevait d'ailleurs tout cela en 2006. Et inversement, de manière moins négative, la recherche scientifique a aussi travaillé sur les facteurs de résilience face à l'homophobie. Ainsi, nous savons que le soutien de l'environnement joue un rôle clé (voir Chamberland et Petit). Plus encore, rencontrer des pairs, d'autres jeunes LGBT, diminue de façon importante la détresse et les risques de suicide. En 2002, la recherche de Otis, Ryan, Chouinard et Fournia Effets du Projet 10 sur le mieux-être sexuel de jeunes gais, lesbiennes, bisexuelles et bisexuels recommandait l'augmentation du financement de l'organisme sur la base de ses résultats. La CDPDJ et le Conseil permanent de la jeunesse $(\mathrm{CPJ})$ en arrivaient à des recommandations similaires :

Que le [ministère de la Santé et des Services sociaux] MSSS soutienne les organismes communautaires, tels des groupes de discussion, des lieux de socialisation et de loisirs, voués aux jeunes de minorités sexuelles. (CDPDJ, 2007)

2. Aux États-Unis : Tyler Clementi (18 ans, New Jersey), Seth Walsh (13 ans, Texas), Raymond Chase (19 ans, Rhode Island), Billy Lucas (15 ans, Indiana), Asher Brown (13 ans), Jamey Rodmeyer (14 ans, New York). Au Canada : Jamie Hubley (15 ans, Ontario), Chantal Dubé, Jeannine Blanchette. Voir le communiqué de presse du 6 octobre 2010 de PFLAG Canada: [http:www.pflagcanada.ca/pdfs/2010-10-06_suicide-deaths-gayyouth.pdf].Au Québec, la disparition de David Fortin a soulevé de nombreux débats.

3. Reteaching gender and sexuality, disponible en ligne [http://www.reteachinggenderandsexuality.org/].

4. Ce serait également la conclusion de Être homo aujourd'hui en France, de Michel Dorais et Isabelle Chollet. 
Que le gouvernement du Québec assure un financement adéquat aux organismes communautaires de lutte contre l'homophobie qui interviennent dans les écoles ainsi qu'à ceux qui offrent des espaces de loisirs et de socialisation aux jeunes de la diversité sexuelle. (CPJ, 2007)

Dix ans après la recherche de Otis et al., où en sommes-nous? Projet 10, le seul groupe bilingue pour les jeunes LGBT qui soit subventionné par le Programme de soutien aux organismes communautaires (PSOC) à Montréal, a dû déménager deux fois et réduire son personnel à deux employés à temps partiel. Jeunesse Lambda fonctionne depuis vingt-cinq ans avec moins de 5000 \$ de budget par année. Les groupes dans les cégeps ont de la difficulté à survivre plus d'une année suite aux mélodrames internes des « qui a couché avec qui ? » et des «qui ne parle plus à qui ? ». Queer line (de Queer McGill) et Gay Line, tenue par la Canadian Association for Education and Outreach (CAEO), ont arrêté leurs services de ligne d'écoute, laissant un vide au niveau des services en anglais dans la province. La Direction de la santé publique n'a plus subventionné la Table de concertation des jeunes GLB de Montréal, devenue par la suite la Coalition jeunesse montréalaise de lutte à l'homophobie (CJMLH), alors que ses débuts étaient prometteurs. Le Regroupement d'entraide pour la jeunesse allosexuelle du Québec (REJAQ), l'association nationale créée en 2001 et regroupant les organismes œuvrant auprès des jeunes LGBT, est morte faute de financement. Quant aux régions, certaines sont mieux desservies que Montréal tandis que d'autres n'ont encore aucun groupe. À notre connaissance, les groupes pour jeunes LGBT dans le reste du Québec sont : Jeunesse Idem (Gatineau), Le Néo (Terrebonne), l'Accès (Québec), Divers-Gens (Granby), le Jeunes Adultes Gai(e)s (JAG) (Saint-Hyacinthe) et le LGBTQ Youth Center (Beaconsfield).

\section{LA PAROLE DES JEUNES DANS L'ESPACE PUBLIC : LES LIEUX D'EXPRESSION}

Les groupes pour jeunes LGBT représentent pourtant un potentiel de transformation sociale important. En effet, ce sont des espaces où ces jeunes, en se réunissant, peuvent prendre conscience de la réalité d'autrui. II ne s'agit pas seulement de briser l'isolement au niveau personnel, mais également au niveau communautaire et politique. Car il existe peu de lieux dans la communauté gaie où un ou une jeune peut venir échanger de manière relativement publique sur son vécu et faire connaître ses difficultés. Faisons rapidement le tour de quelquesuns de ceux-ci.

Il y a d'abord les bars et le Village gai. Si ceux-ci ont joué un rôle important dans la socialisation des personnes LGBT et la construction d'une résistance aux oppressions qu'elles vivent (pensons à Stonewall, au Truxx et au Sex Garage), ils demeurent des lieux commerciaux, orientés vers la consommation, et commandent des interactions basées principalement sur le désir. Nous pourrions d'ailleurs noter la forte division selon le genre et l'âge qui s'applique dans ces établissements ${ }^{5}$. L'idée n'est pas de rejeter en bloc ces espaces; il s'y développe après tout de nombreuses amitiés et de nouvelles solidarités comme le rappelle le collectif queer Trouble dans son énoncé de mission: on y affirme que la communauté se

5. Il aura fallu attendre 2008 pour que la CDPDJ intervienne dans le cas de la non-mixité de certains bars gais. Cette situation n'a cependant pas été révoquée dans les saunas. Voir le communiqué de la CDPDJ : [http://www.cdpdj.qc.ca/comm/COMM_BAR_LE_STUD_reglement.pdf]. 
construit sur la piste de danse ${ }^{6}$. Trouble est, jusqu'à présent, un groupe organisant des soirées dansantes alcoolisées qui servent de levées de fonds pour Aids Community Care Montreal (ACCM). Entre Trouble et les bars, il existe cependant toute une différence : la « communauté » a davantage son mot à dire sur la façon dont le premier s'organise, tandis qu'elle n'a que peu d'influence sur les décisions des deuxièmes, axés sur la rentabilité de leur commerce. II y a moyen pour un individu de s'impliquer dans l'organisation des soirées de Trouble (ou dans celles similaires de nombreux collectifs queers tels que la Radical Queer Semaine ou Pervers/Cité). Une attention particulière est mise sur la question de l'accessibilité - avec des niveaux de réussite variable, du fait notamment que la plupart de ces lieux demeurent des espaces réservés aux 18 ans et plus.

Les sites de rencontre en ligne, tels Gay411, ou OKCupid, sont des lieux d'échanges individuels souvent orientés par une dynamique de séduction, similaire en cela aux bars (et souvent tout aussi séparé sur le plan des genres). Ces sites sont de merveilleux terrains de recherche sur les perceptions et représentations qui circulent dans la communauté gaie. On peut dire, à partir du langage qui s'y est développé (Party and Play [PNP], BB, clean, hors milieu, etc.), qu'il y a là de véritables cultures virtuelles. L'appropriation de ces codes par une personne qui découvre son orientation sexuelle et qui s'inscrit à de tels sites nécessite un certain apprentissage. Cela peut expliquer que plusieurs utilisateurs n'hésiteront pas à faire preuve d'ironie face à ce médium, et ce, directement sur leur profil de présentation. II faut dire que les interactions virtuelles sont pour la plupart du temps individuelles, médiatisé par le site qui en délimite les usages, les catégories sous lesquelles s'identifier et les conditions d'accès (payant ou non). Ces sites empêchent ainsi une prise de parole collective qui permettrait à ceux éprouvant une aliénation face à ces normes d'agir sur celles-ci. Mais ils permettent aux utilisateurs de se rencontrer sans avoir à se déplacer et sans avoir à entrer en contact avec un groupe. Chacun y est libre de déterminer ce qu'il cherche, sans jugement collectif.

Le monde virtuel ne se limite cependant pas aux seuls sites de rencontre puisqu'on y retrouve aussi de nombreux forums autogérés (Alterhéros, Ado Gay Québec, etc.). Ceux-ci se rapprochent sans doute le plus des espaces de socialisation politique dont nous parlons, par leur capacité à rendre visible à tous le vécu d'autrui et à permettre l'organisation collective. Car on retrouve de tout sur les forums: témoignages, discussions, échange de photos, etc. Leurs avantages sont de rejoindre les personnes éloignées géographiquement et de leur permettre de garder l'anonymat. Ce constat ne remplace cependant pas le besoin d'interactions réelles dans l'environnement immédiat de ces personnes, d'où l'importance de groupes pour jeunes LGBT.

6. Voir le facebook du collectif : [https://www.facebook.com/pages/Collectif-Trouble-Collective/282179551899881]. 


\section{L'APPORT DES GROUPES JEUNESSE LGBT AU QUÉBEC : FRAGMENTS D'HISTOIRE}

Certains groupes jeunesse LGBT ont des réalisations plutôt impressionnantes à leur actif. Nous aimerions en donner quelques exemples afin de laisser quelques traces sur leur histoire.

Le plus frappant est sans doute le groupe de discussion Jeunesse Lambda. L'organisme est géré depuis plus de vingt-cinq ans par et pour des jeunes gais, lesbiennes et bisexuel(le)s de moins de 25 ans (presque exclusivement). Sa création découle de Lambda Youth : ayant vu le jour sous l'égide du Ville-Marie Social Work, maintenant devenu les Centres de la famille Batshaw, il s'agit d'une initiative anglophone pour les jeunes de 14 à 21 ans. Très rapidement, le groupe se sépare de son port d'attache. Comme le rapporte Carle Bernier-Genest dans son historique de Jeunesse Lambda, les organisateurs tentent de mettre en place des soirées francophones, mais celles-ci échouent et l'anglais domine toujours les discussions ${ }^{7}$. C'est pourquoi plusieurs décident de fonder la version française du groupe. Lambda Youth n'y survivra que peu de temps et ferme vers 1989.

Dès ses débuts, l'organisme se donne trois missions : briser l'isolement des jeunes LGB; accompagner ces jeunes dans leur acceptation de leur orientation sexuelle; démystifier les réalités de la diversité sexuelle. En plus d'organiser leurs soirées de discussion du vendredi, les jeunes écrivent un journal, planifie des activités sociales et parfois provocantes (une anecdote de leur journal rapporte une soirée à la cabane à sucre plutôt animée), tiennent des stands dans divers événements d'envergure (dont le Salon Pepsi-jeunesse), participent à des tables de concertation (dont la Table de concertation des lesbiennes et des gais du Grand Montréal et le Comité de défense juridique) et marchent fièrement à Divers/Cité. Leur cinquième anniversaire attira plus de 500 personnes. C'est cependant des groupes qu'il engendra que nous désirons parler. Notons, dans un premier temps, Aux Prismes, un club d'activités sociales qui lui-même engendra celui de plein air Hors sentiers. Ce furent aussi des personnes de Jeunesse Lambda qui firent les premières démystifications dans les écoles et créèrent le Comité d'interventions sociales. Celui-ci, prenant de plus en plus d'ampleur au sein de l'organisme, se sépara pour devenir le Groupe de recherche et d'intervention sociale-Montréal, mieux connu sous l'appellation GRIS-Montréal (qui lui-même inspira la structure des autres GRIS à travers le Québec).

On retrouve encore beaucoup de similitudes de structure entre Jeunesse Lambda et le GRIS-Montréal. Les plus importantes sont l'engagement des membres à tous les niveaux de l'organisme et l'utilisation du vécu individuel comme outil de changement social. Ce qui diffère, c'est l'évolution du GRIS-Montréal après sa séparation de Jeunesse Lambda. En effet, le GRISMontréal s'est développé de manière exponentielle. Grâce à plus de 200 bénévoles, il réussit à rejoindre près de 20000 jeunes par année. Sa dernière campagne de financement a amassé plus de 170000 \$. L'organisme a reçu de nombreux prix, dont un du réseau de la santé. II est sollicité par plusieurs instances et ministères pour son expertise. Qu'est-ce qui fait que le GRISMontréal a pu se développer et pas Jeunesse Lambda? D'abord la question de l'autonomie et

7. Pour l'historique complet, voir : [http://jeunesselambda.org/information]. 
du réseau social des membres du GRIS. Plus âgés, ayant fait leur coming-out, ils sont mieux établis socialement. Ils peuvent facilement solliciter leur entourage, clé du succès des organismes communautaires qui repose sur l'investissement des gens. À l'inverse, les animateurs de Lambda doivent souvent jongler entre leur emploi étudiant et leur curriculum scolaire, en quête identitaire, tout en tâchant de se faire un réseau d'amis. Lambda est presque en soi une école: on y apprend à gérer un groupe, on y découvre les rouages de la bureaucratie et on y fait l'expérience des conflits. Le manque de ressources n'est donc pas le fait d'un manque d'organisation, mais bien d'un enjeu structurel lié à l'âge de ses organisateurs qui les place dans des situations parfois précaires.

Une autre initiative importante qui illustre cette situation est la durée de vie du REJAQ. Créé en 2001, sa visée était de « doter l'ensemble du mouvement de la jeunesse allosexuelle du Québec d'une structure lui permettant d'échanger, partager ses préoccupations et faire évoluer les dossiers d'ordre national ». Le REJAQ a organisé durant sept ans des rencontres semestrielles bisannuelles de formation et d'échanges entre les groupes jeunesse LGBT du Québec. Il s'est réapproprié le mot « allosexuel » en lui donnant une nouvelle définition, soit la traduction partielle du terme « queer »:

Afin de trouver un mot unificateur du concept de la diversité sexuelle, le REJAQ a repris le mot «allosexuel» pour y désigner toute personne éprouvant des attirances sexuelles et étant confrontée à celles-ci, à de la discrimination ou à des questionnements face à leur orientation sexuelle ou à leur identité de genre. Ce néologisme, désormais reconnu par l'Office de la langue française, inclut donc les personnes gaies, lesbiennes, bisexuelles, transgenres, transsexuelles ou bispirituelles. Étant le contraire d'une étiquette, ce terme est de plus en plus utilisé par les membres de notre regroupement ${ }^{8}$.

Si on peut considérer que le terme n'a eu que peu de succès sur le terrain, les jeunes ne s'y reconnaissant pas, il a tout de même circulé dans les milieux universitaires et politiques. II n'est en effet pas rare de voir des questionnaires de recherche utiliser « allosexuel » comme choix de réponse à la catégorie d'auto-identification de l'orientation sexuelle. Plus d'une vingtaine de groupes ont utilisé l'appellation et quelques rapports institutionnels l'ont aussi reprise ${ }^{9}$. Le poste réservé aux personnes gaies et lesbiennes au conseil des jeunes élus du Forum jeunesse de l'île de Montréal porte toujours le nom de siège allosexuel. C'est que le REJAQ a très rapidement su se faire reconnaître en écrivant des mémoires et en devenant membre de la Table des regroupements provinciaux d'organismes communautaires et bénévoles (TRPOCB). Il fut d'ailleurs le représentant jeunesse au Comité de travail mixte ayant mené au rapport De l'égalité juridique à l'égalité sociale - Pour une politique nationale de lutte à l'homophobie de la CDPDJ. Afin de reconnaître l'apport des jeunes de la diversité sexuelle dans la société, il met sur pied le Gala des Allostars qui, trois ans durant, saluera les individus, les groupes et les

8. Regroupement d'entraide pour la jeunesse allosexuelle du Québec (2005) Mémoire, mémoire déposé dans le cadre du renouvellement de la Stratégie action jeunesse 2005-2008 du Gouvernement du Québec. Disponible en ligne : [http:www.conseil-Igbt.ca/uploads/files/regroupement-entraide-pour-la-jeunesse-allosexuelle-duquebe.pdf].

9. Voir notamment les mémoires des Forums jeunesse lors du renouvellement de la Stratégie action jeunesse 2005-2008. 
parents ayant contribué à la lutte contre l'homophobie au Québec. Le Gala deviendra même trop gros (en termes de gestion) et se séparera de l'organisme. Mais celui-ci meurt la même année que le REJAQ. Faute de financement pour faire vivre un organisme national (ne serait-ce que pour assumer le coût des déplacements et des appels interurbains), le regroupement sera dissous à contrecœur par ses membres.

\section{CONSÉQUENCES DU MANQUE DE RESSOURCE SUR L'ORGANISATION DES SERVICES SPÉCIALISÉS}

Nous aimerions maintenant discuter des conséquences du manque de ressources des groupes sur les capacités d'intervention du réseau.

1) D'abord, les intervenants des milieux de la santé et de l'éducation connaissent peu les ressources pour les jeunes LGBT et les réseaux d'entraide qui se forment. Déjà en 2002, une recherche de Beaulieu et Martin auprès d'enseignants de la Commission scolaire de Montréal notait que «76\% se disent peu ou très peu informés sur les réalités homosexuelles » et que « $74 \%$ disent avoir besoin d'information ou de formation ». En 2007, le Conseil permanent de la jeunesse (CPJ) a également interrogé les professionnels du milieu scolaire sur leur capacité à intervenir auprès des jeunes de la diversité sexuelle. Dans son rapport Sortons l'homophobie du placard... et de nos écoles secondaires, le CPJ recense que $45 \%$ des répondants sont peu capables d'identifier des ressources. Et les moyens les plus fréquemment cités sont des dépliants et des affiches. II souligne que « cette proportion est suffisamment grande pour mettre en doute la connaissance qu'a le personnel scolaire des outils d'information et de sensibilisation sur la diversité sexuelle, de même que la disponibilité de ces outils »(CPJ, 2007, p. 60). Si seulement $67 \%$ des répondants considèrent que leur personnel est outillé au sujet de la diversité sexuelle, $94 \%$ d'entre eux affirment pouvoir rediriger un jeune se questionnant sur son identité sexuelle vers une personne compétente. II nous semble y avoir là une contradiction.

2) À la défense des intervenants, il faut reconnaître que plusieurs groupes, notamment ceux dans les cégeps ou les petits collectifs, n'ont pas la stabilité (financière, humaine) qui leur assurerait une pérennité. Cela affecte à la fois la capacité des organismes à se faire connaître (puisqu'il y faut du temps) et à offrir des services. La mémoire institutionnelle de ces groupes est souvent perdue avec le départ des individus. De plus, le grand roulement des membres responsables (en termes juridiques comme membres du conseil d'administration ou, de façon informelle, comme membres du collectif) rend difficile d'identifier une personne-contact. C'est par le bouche-à-oreille que l'on peut connaître l'existence d'une ressource et savoir si elle opère encore. Les professionnels du réseau de la santé ne font généralement pas partie des cercles sociaux des jeunes où se transmet ce genre d'information. Leur pratique ne leur permet pas de maintenir le réseau nécessaire pour cela. II faudrait une ressource pour arriver à compiler cela. C'est ce que tente de faire la CJMLH, elle-même sans financement pour répondre à ce défi. 
3) Les organismes bénéficiant d'un financement suffisant pour avoir des permanents demandent une grande polyvalence à leurs employés. La réalité, du moins à Montréal, c'est que ces derniers doivent s'y connaître en matière d'itinérance, de toxicomanie, de violence conjugale, de transition de genre, de suicide, d'automutilation, d'immigration, de système de protection de la jeunesse, de santé sexuelle et d'ITSS, de droit du logement, de réinsertion au travail, de santé mentale, et ce, bien souvent, sans avoir accès aux corridors de service des Centres de santé et de services sociaux (CSSS). À cela s'ajoute tout le travail administratif (recherche de subvention, comptabilité, etc.), de sensibilisation (ateliers, formations, conférences, etc.), d'encadrement (stagiaires, bénévoles, conseil d'administration) et de communication (promotion, réseautage, etc.) qui retombe sur ces mêmes personnes. Tel que mentionné plus haut, Projet 10 a deux employés à temps partiel et Jeunesse Lambda n'a aucun permanent. II y aurait donc à réfléchir sur les conditions de travail des employés des groupes LGBT et le soutien à fournir pour leur éviter le burn-out.

4) Dans le milieu de la santé, il est souvent question de corridors de service. On désigne ainsi les processus mis en place pour favoriser la référence entre services médicaux ou sociaux afin qu'une personne aux prises avec de multiples problématiques puisse être prise en charge par divers spécialistes, sans devoir faire elle-même toutes les démarches de manière isolée, en risquant de ne pas trouver le bon service ou de se perdre dans les dédales administratifs et en risquant de ne pas obtenir l'accès aux soins dont elle a besoin. Il s'agit de prendre charge de la santé de la personne de manière holistique et de voir comment un enjeu est souvent relié à un autre.

Considérant la multitude des besoins énoncés plus haut, les intervenants ont aussi du mal à trouver des ressources externes où les jeunes pourront être assurés qu'ils ne vivront pas d'homophobie ou de transphobie. Nous avons commencé à développer une banque de professionnels, notamment en ce qui a trait aux personnes trans. Mais la plupart de ces professionnels ont des pratiques privées et demeurent peu accessibles financièrement pour des jeunes aux revenus précaires.

5) On devrait établir un meilleur suivi entre les groupes jeunesse LGBT et les intervenants qui réfèrent des jeunes. II n'est pas rare qu'un intervenant avec un jeune qui aurait, par exemple, un syndrome d'Asperger, le réfère à un organisme comme Projet 10 ou Jeunesse Lambda sans établir de lien préalable. Si nos groupes peuvent permettre de briser l'isolement lié à l'orientation sexuelle, ils n'ont pas les moyens de faire un suivi à moyen et long terme pour agir sur les enjeux de santé globale affectant ces jeunes, notamment quand il y a des problématiques entrecroisées. II faut une équipe capable de faire le pont entre les divers aspects de la vie de cette personne. Un meilleur suivi des intervenants serait apprécié (des rencontres conjointes avec le travailleur social du jeune ou l'intervenant de son école seraient à envisager). Cette approche a bien sûr ses limites, puisque l'on veut respecter la confidentialité du jeune lorsqu'il est à l'organisme et son autonomie à prendre ses propres décisions. Cependant, l'arrivée d'un participant dans un groupe sans permanent et sans intervenant formé en santé mentale peut avoir des conséquences lourdes tant sur le jeune qui n'arrive pas toujours à s'intégrer par lui-même, que sur le groupe qui peut risquer de se dissoudre due à la lourdeur du soutien nécessaire ou qui 
risque de prendre des décisions collectives pour survivre au détriment du participant. II est donc crucial de subventionner adéquatement les groupes pour jeunes, afin de leur permettre de maintenir une formation permanente capable de faire face aux multiples dimensions du travail social. Bien sûr, il faudrait pouvoir compter sur les intervenants jeunesse, puisque c'est aussi leur tâche (et plusieurs sont déjà de précieux alliés). Mais ceux-ci sont très peu formés pour intervenir dans la lutte à l'homophobie.

Bruno Laprade

Coordonnateur de Projet 10

\section{BIBLIOGRAPHIE}

Otis, J., B. Ryan, N. Chouinard et K. Fournia (2002). Effets du Projet 10 sur le mieux-être sexuel de jeunes gais, lesbiennes, bisexuelles et bisexuels. Rapport de recherche subventionnée par la Régie régionale de la santé et des services sociaux de Montréal Centre.

Commission des droits de la personne et des droits de la jeunesse (2007). De l'égalité juridique à l'égalité sociale - vers une stratégie nationale de lutte contre l'homophobie. Rapport de consultation du Groupe de travail mixte contre l'homophobie. Québec.

Conseil permanent de la jeunesse (2007). Sortir l'homophobie du placard... et de nos écoles secondaires. Rapport déposé au Secrétariat à la jeunesse.

Dorais, Michel et Isabelle Chollet (2012). Être homo aujourd'hui en France. Saint-Martin-deLondres : H \& O, collection « Essais ».

Regroupement d'entraide pour la jeunesse allosexuelle du Québec (2005). Mémoire, mémoire déposé dans le cadre du renouvellement de la Stratégie action jeunesse 2005-2008 du Gouvernement du Québec. 